\title{
DIAGNOSTICS
}

\section{LCR testing for gonorrhoea and chlamydia in population surveys and other screenings of low prevalence populations: coping with decreased positive predictive value}

\author{
J M Zenilman, W C Miller, C Gaydos, S M Rogers, C F Turner
}

Sex Transm Infect 2003;79:94-97

\begin{abstract}
Objective: Nucleic acid amplification tests have facilitated field based STD studies and increased screening activities. However, even with highly specific tests, the positive predictive value (PPV) of such tests may be lower than desirable in low prevalence populations. We estimated PPVs for a single LCR test in a population survey in which positive specimens were retested.

Methods: The Baltimore STD and Behavior Survey (BSBS) was a population based behavioural survey of adults which included collecting urine specimens to assess the prevalence of gonorrhoea and chlamydial infection. Gonorrhoea and chlamydial infection were diagnosed by ligase chain reaction (LCR). Nearly all positive results were retested by LCR. Because of cost considerations, negative results were not confirmed. Predicted curves for the PPV were calculated for a single testing assuming an LCR test sensitivity of $95 \%$, and test specificities in the range $95.0 \%-99.9 \%$, for disease prevalences between $1 \%$ and $10 \%$. Positive specimens were retested to derive empirical estimates of the PPV of a positive result on a single LCR test. Results: 579 participants age 18-35 provided urine specimens. $20(3.5 \%)$ subjects initially tested positive for chlamydial infection, and $39(6.7 \%)$ tested positive for gonococcal infection. If positive results on the repeat LCR are taken as confirmation of a "true" infection, the observed PPV for the first LCR testing was $89.5 \%$ for chlamydial infection and $83.3 \%$ for gonorrhoea. This is within the range of theoretical PPVs calculated from the assumed sensitivities and specificities of the LCR assays.

Conclusions: Empirical performance of a single LCR testing approximated the theoretically predicted PPV in this field study. This result demonstrates the need to take account of the lower PPVs obtained when such tests are used in field studies or clinical screening of low prevalence populations. Repeat testing of specimens, preferably with a different assay (for example, polymerase chain reaction), and disclosure of the non-trivial potential for false positive test results would seem appropriate in all such studies.
\end{abstract}

See end of article for authors' affiliations

Correspondence to: Jonathan M Zenilman, Infectious Diseases Division, John Hopkins University School of Medicine, Baltimore, MD USA; jzenilma@jhmi.edu

Accepted for publication 17 October 2002

C hlamydial infection and gonorrhoea are the two most common bacterial sexually transmitted infections in the United States, ${ }^{1}$ predominantly affecting adolescents and young adults. Medical and public health interventions require accurate diagnosis, which is easily accomplished for symptomatic patients. Most gonococcal and chlamydial infections in women are asymptomatic. In male clinic populations, the literature suggests that up to $10 \%$ of gonococcal urethritis, ${ }^{23}$ and a third of chlamydial urethritis may be asymptomatic. ${ }^{4}$ In population based surveys, however, the proportion of asymptomatic disease is higher. Screening asymptomatic people at risk for infection has therefore evolved as a major public health control strategy.

New non-invasive nucleic acid amplification tests (NAATs), such as ligase chain reaction (LCR), have been licensed for diagnosis of gonorrhoea and chlamydial infection in urine for both males and females. ${ }^{5-8}$ Testing of urine specimens eliminates the need for clinical examination, and it provides the opportunity for STD screening in community based sites, such as schools, ${ }^{9}$ military field clinics, ${ }^{10}$ developing country settings, ${ }^{11}$ and primary care clinics. Non-invasive screening can also be used in population surveys, such as the ones recently performed in Baltimore, ${ }^{12}$ in the National Survey of Adolescent Males, ${ }^{13}$ in a pilot test of the National Health and
Nutrition Examination Survey, ${ }^{14}$ and in a study of Job Corps participants. ${ }^{15}$

Use of NAAT results for STD diagnosis in population surveys or other screenings of low prevalence populations is associated with a lower positive predictive value (PPV) and higher proportion of false positive results than one encounters in clinical practice. This issue has been previously raised by Schachter and Chow. ${ }^{16}$

In this paper, we report on our experience conducting a population survey that employed LCR tests to diagnose gonococcal and chlamydial infections. We estimated PPVs based on a single LCR test and compared the theoretically predicted PPV for a single testing with the results we obtained when positive LCR tests were retested for confirmation. Discordance in test results across these two testings provides an empirical measure (albeit an imperfect one) of the predictive value of a positive result in a single LCR testing.

\section{METHODS}

Baltimore STD and Behavior Survey

The Baltimore STD and Behavioral Survey (BSBS) was a population based cross sectional household survey of adults residing in Baltimore, MD, USA. Survey interviews were conducted with 1014 respondents ages 18-45 years between 
January 1997 and September 1998. According to the study protocol, only respondents between 18 and 35 years of age were asked to provide a urine specimen for gonorrhoea and Chlamydia trachomatis testing. Of the 728 respondents aged $18-35,579(80 \%)$ provided a urine sample, $16 \%$ refused, and $4 \%$ were not tested (owing to interviewer error, inability to provide a specimen, insufficient volume, etc).

For study purposes, respondents were considered positive if both an initial and a confirmatory LCR were positive. Repeat tests were unavailable for three cases who tested positive initially. These cases were classified as positive based on their first testing. All respondents were given a telephone number they could call to learn of their test results. Study staff used a succession of methods to attempt to contact participants who were confirmed positive (telephone, registered letter, and, if refused or undelivered, regular mail). Free, expedited treatment at one of the Baltimore City Department of Health clinics was offered to all contacted subjects with confirmed positive results.

Details of the full study design have been described elsewhere. ${ }^{12}$ Protocols were approved by the institutional review board at Research Triangle Institute and the Johns Hopkins University.

\section{LCR assays}

\section{First LCR testing}

Urine testing was performed following standard LCR procedures detailed by the manufacturer. (LCX-Abbott Laboratories, Abbott Park, IL, USA). For Neisseria gonorrhoeae, the optical density (OD) cut offs for the LCR products were: negative $=$ $<0.8$; indeterminate: $0.8<x<1.2$; positive $>1.2$. For the $C$ trachomatis assay, negative results were defined as less than 0.8 , indeterminate: $0.8<x<1.0$, and positive as $>1.0$. All tests in the indeterminate range were retested to provide a "first test" result.

\section{Second testing}

We retested 19 of 20 specimens that tested positive for chlamydial infection on their first LCR testing and 36 of 39 specimens that tested positive for gonococcal infection. Two specimens that initially tested positive for gonococcal infection and one specimen that initially tested positive for both gonococcal and chlamydial infections were not retested because the original specimen was not available. We defined a confirmed positive result as a specimen with positive results on both initial and repeat testing. Negative tests were not confirmed.

\section{Theoretical expectations for positive predictive value}

The positive predictive value, which reflects the post-test probability of disease for a positive test ${ }^{17}$ is defined as:

$\mathrm{PPV}=$ probability (disease/test positive) $=$ true positives/ (true positives + false positives)

The positive predictive value can also be expressed in a form based on Bayes's theorem ${ }^{17}$ :

$\mathrm{PPV}=($ sensitivity $\times$ prevalence $) /($ sensitivity $\times$ prevalence $)$ $+(1-$ specificity $) \times(1-$ prevalence $))$

This expression of PPV highlights the dependence of the PPV on prevalence and permits the prediction of PPV across a range of prevalences for a test with known sensitivity and specificity.

Previous work from our group has reported sensitivities of urine LCR testing in our laboratory of $88.6 \%$ to $95.5 \%$, and specificity of $99 \%$ for women. ${ }^{18}{ }^{19}$ Given the dependence of PPV on the prevalence and specificity, plots were constructed of the predicted PPV across a range of plausible values for prevalence $(0.5 \%-10 \%)$ and specificity $(95.0 \%-99.9 \%)$. The specificity estimates represent a range that we would expect when the tests are used in a variety of field settings outside the research

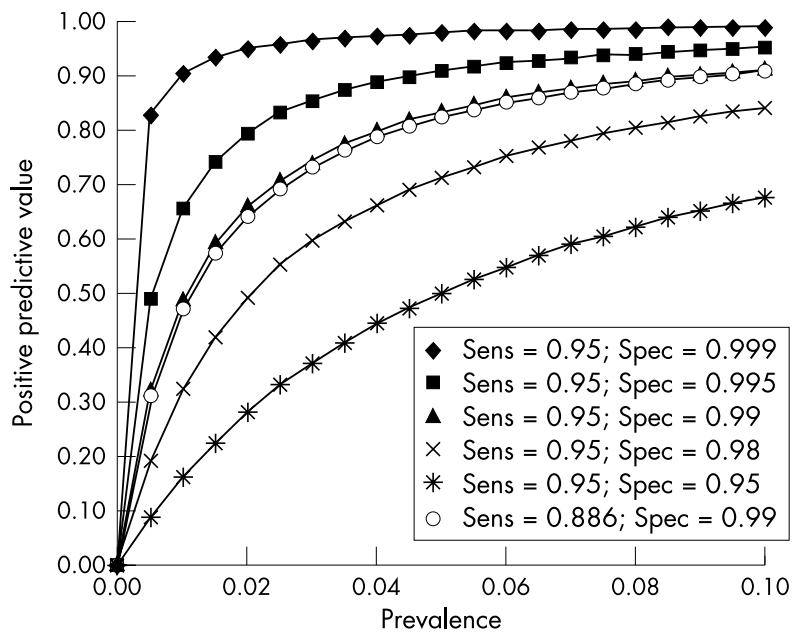

Figure 1 Calculated positive predictive values for a test with sensitivity of $88.6 \%-95 \%$ and specificity of $95 \%-99.9 \%$ in theoretical populations with disease prevalences of $0.0 \%-10.0 \%$. Note steep decrease in PPV at lower prevalence levels.

laboratory. We examined PPV at a sensitivity of $95.0 \%$ and $0.88 .6 \%$, but the effect of sensitivity on PPV was small. These sensitivity estimates were used because they represent the lower bounds of LCR sensitivity for diagnosis from urine in earlier studies.

We compared the theoretically derived positive predictive value of a single LCR result with the empirically observed PPV, using a reference standard of "confirmed positive results" (that is, positive on both first and second testing).

\section{RESULTS}

\section{Theoretical expectation}

Across a range of plausible prevalences and test characteristics, the theoretical expectation for PPV of a single LCR testing remained relatively low ( fig 1). At 99.0\% specificity and $95.0 \%$ sensitivity, the PPV was $66 \%$ if population prevalence was $2 \%$, and it dropped to $32 \%$ if population prevalence was $0.5 \%$. Even if specificity were increased to $99.9 \%$, PPV at $2 \%$ prevalence was $95.1 \%$, and at $0.5 \%$ prevalence it was $82.6 \%$; a result that predicts nearly one in five positive test results will be false positive if true prevalence were as low as $0.5 \%$. (Similar results are obtained if sensitivity is assumed to be $88.6 \%$ instead of $95 \%$; see results in fig 1.)

\section{Empirical observations}

In our field study, there were 579 eligible participants. Twenty $(3.5 \%)$ subjects initially tested positive for chlamydial infection and $39(6.7 \%)$ tested positive for gonococcal infection. Retesting was performed on 19 of 20 positive chlamydia specimens and 36 of 39 positive gonorrhoea specimens. (No retests were available for two specimens that initially tested positive for gonorrhoea and one specimen that tested positive for both gonorrhoea and chlamydial infection.) Retests yielded a second positive result in 17/19 (89.5\%) cases of chlamydial infection and 30/36 (83.3\%) gonorrhoea cases. These empirical results indicate that the positive predictive values of the first LCR testing in our study were $89.5 \%$ for chlamydial infection and $83.3 \%$ for gonorrhoea.

The unweighted prevalence of confirmed infections was $17 / 576(3.0 \%)$ for chlamydial infection and 30/576 (5.2\%) for gonococcal infections. (The unweighted sample counts represent the results of our NAAT testing, they do not, however, provide valid estimates of the prevalence of infection in the population as a whole or in any subpopulation. Since we used 
Table 1 Calculated PPV for theoretical populations with of prevalence of $0.5 \%-10 \%$ and test specificity of $98 \%-99.5 \%$

\begin{tabular}{lllll}
\hline Prevalence & PPV at 98\% Spec & PPV @ 99\% Spec & PPV @ 99.5\% Spec & PPV @ 99.9\% Spec \\
\hline $10 \%$ & 84.1 & 91.3 & 95.4 & 99.1 \\
$5 \%$ & 71.4 & 83.3 & 90.1 & 98.0 \\
$2 \%$ & 49.2 & 65.9 & 79.4 & 95.1 \\
$1 \%$ & 32.4 & 48.9 & 65.7 & 90.5 \\
$0.5 \%$ & 19.2 & 32.3 & 48.8 & 82.6 \\
\hline
\end{tabular}

a complex sample design which purposely oversampled certain segments of the population (see Turner et $a l^{12}$ ), only the weighted estimates can be used to make inferences about the prevalence of NAAT detectable infections in the population.) Assuming specificities for a single LCR testing to be 99.0\%-99.6\%, the theoretically expected PPV for a single LCR testing for chlamydia would be $73 \%-87 \%$, and for gonorrhoea it would be $82 \%-92 \%$. This theoretical expectation is in agreement with our empirical results.

\section{DISCUSSION}

The availability of new, non-invasive NAATs (for example, LCR and polymerase chain reaction (PCR)) for STDs has resulted in recommendations to extend screening to venues of moderate and low prevalence such as emergency departments, primary care clinics, and others. Chlamydia testing is now recommended as part of routine care for sexually active women under 25 years of age. Using clinical tests to screen low prevalence populations presents new issues in the case of STD testing. A positive STD test result has implications for treatment, but it may also lead subjects to speculate about the source of their infection. This speculation could lead to social or psychological stress, especially if the individual is in a perceived monogamous relationship. Research and screening programmes that test low prevalence populations must reduce both the incidence of false positives and ameliorate their consequences.

Even under optimal conditions, unless the test specificity is $100 \%$, clinicians will face the "epidemiological brick wall" described in figure 1 and table 1 . These false positive results are predicted by Bayes's theorem and are statistically unavoidable unless test specificity is $100 \%$, which is seldom, if ever, attainable. Testing clinical specimens requires execution of a series of steps including specimen collection, transport, processing and detection, each of which is subject to error. In the BSBS survey, 36 field interviewers were employed to reach a population based sample of Baltimore City residents. Considering the complexity of the logistics, we were pleased with the diagnostic test's performance on a single testing. The number of false positives that we observed in this study using repeat LCR testing were predictable based on test characteristics and the low prevalence of infection in the population.

\section{Limitation of study}

Our estimates of the PPV of LCR testing for gonococcal and chlamydial infection are limited by the absence of an independent "perfect" reference standard. We used as a reference standard a "confirmed positive result" based on initial and repeat LCR testing using the same specimen and the same test procedure. The use of the same test on the same specimen has the potential to misclassify some positive results, because a false positive test may be repeatedly false positive. Therefore, our empirical determination of the PPV for a single LCR testing might be viewed as the upper limit of the PPV for these tests because a small number of false positive results could go undetected with this testing scheme.

\section{Recommendations}

When clinical decisions are to be made based on test results from the screening of a low prevalence population, we believe that confirmatory algorithms are necessary. Repeat testing of positives will increase specificity and reduce the incidence of false positive test results. Ideally, a different, highly specific and sensitive test should be used as the confirmation. An initial testing with LCR might, for example, be confirmed by a repeat assay with PCR.

Since no testing protocol will completely eliminate the threat of false positives, it will also be important to inform subjects with confirmed positive test results of the possibility of test error. This is a tricky task since we do not wish to discourage subjects from seeking treatment. In the BSBS, we eventually settled upon a script that:

(1) informed subjects that the tests are approved by the FDA and have been found to be very reliable, but there was always the possibility for error with any test; and

(2) if the respondent had doubts about the test result, offered-at study expense-to collect another urine specimen and test it.

For all respondents except those who requested additional testing, we emphasised the importance of obtaining treatment and, as before, provided them with the address of the Baltimore public health clinic that would provide free, expedited treatment, if they did not have a private physician they wished to use.

\section{ACKNOWLEDGEMENTS}

The authors wish to thank Jeff Yuenger for his assistance in organising the laboratory testing and the survey operations staff of the Research Triangle Institute for their fielding of the survey.

Funding/support: Primary support for the Baltimore STD and Behavior Survey (BSBS) was provided by NIH grant R01-HD31067 to Dr Turner. Additional support was provided by the Research Triangle Institute and by grants R01-MH56318 to Dr Turner, K24-AI01633 and U19-AI38533 to Dr Zenilman. Dr Miller received support through the Clinical Associate Physician Program of the General Clinical Research Center (RR00046), Division of Research Resources, National Institutes of Health. Abbott Labs donated some of the LCR test kits used in this study.

Authors' affiliations

J M Zenilman, C Gaydos, Infectious Diseases Division Johns Hopkins University School of Medicine, Baltimore, MD, USA

W C Miller, Division of Infectious Diseases, Department of Medicine and Department of Epidemiology, University of North Carolina at Chapel Hill, Chapel Hill, NC, USA

S M Rogers, C F Turner, Program in Health and Behavior Measurement, Research Triangle Institute, Washington DC, USA

C F Turner, City University of New York, Queens College, and the

Graduate Center, NY, USA 


\section{REFERENCES}

1 Centers for Disease Control. Sexually transmitted disease surveillance, 2000. Atlanta, GA:Centers for Disease Control and Prevention, US Department of Health and Human Services, 2001

2 Daker-White G, Barlow D. Heterosexual gonorrhoea at St Thomas'-I: Patient characteristics and implications for targeted STD and HIV prevention strategies. Int J STD AIDS 1997;8:32-5.

3 Handsfield HH, Lipman TO, Harnisch JP, et al. Asymptomatic gonorrhea in men. Diagnosis, natural course, prevalence and significance. N EnglJ Med 1974;290:117-23.

4 Potterat JJ, Rothenberg RB, Muth SQ. Network structural dynamics and infectious disease propagation. Int J STD AIDS 1999;10:182-5

5 Black CM. Current methods of laboratory diagnosis of Chlamydia trachomatis infections. Clin Microbiol Rev 1997;10:160-84.

6 Chernesky MA, Lee H, Schachter J, et al. Diagnosis of Chlamydia trachomatis urethral infection in symptomatic and asymptomatic men by testing first-void urine in a ligase chain reaction assay. J Infect Dis 1994:170:1308-11

7 Lee HH, Chernesky MA, Schachter J, et al. Diagnosis of Chlamydia trachomatis genitourinary infection in women by ligase chain reaction assay of urine. Lancet 1995;345:213-16.

8 Schachter J, Moncada J, Whidden R, et al. Noninvasive tests for diagnosis of Chlamydia trachomatis infection: application of ligase chain reaction to first-catch urine specimens of women. J Infect Dis 1995; 172:1411-14

9 Burstein GR, Gaydos CA, Diener-West M, et al. Incident Chlamydia trachomatis infections among inner-city adolescent females. JAMA 1998;280:521-6.

10 Gaydos CA, Howell MR, Quinn TC, et al. Use of ligase chain reaction with urine versus cervical culture for detection of chlamydia trachomatis in an asymptomatic military population of pregnant and nonpregnant females attending Papanicolaou smear clinics. J Clin Microbiol 1998;36:1300-4.

11 Paxton LA, Sewankambo N, Gray R, et al. Asymptomatic non-ulcerative genital tract infections in a rural Ugandan population. Sex Transm Infect 1998;74:421-5.

12 Turner CF, Rogers SM, Miller HG, et al. Untreated gonococcal and chlamydia infection in a probability sample of Baltimore adults. JAMA 2002;287:726-33

$13 \mathrm{Ku} \mathrm{L}$, Sonenstein FL, Turner CF, et al. The promise of integrated representative surveys about sexually transmitted diseases and behavior. Sex Transm Dis 1997;24:299-309.

14 Mertz KJ, McQuillan GM, Levine WC, et al. A pilot study of the prevalence of chlamydial infection in a national household survey. Sex Transm Dis 1998;25:225-8

15 Mertz KJ, Ransom RL, St Louis ME, et al. Prevalence of genital chlamydial infection in young women entering a national job training program, 1990-1997. Am J Public Health 2001:91:1287-90.

16 Schachter J, Chow JM. The fallibility of diagnostic tests for sexually transmitted diseases:the impact of behavioral and epidemiologic studies. Sex Transm Dis 1995;22:191-6.

17 Sackett DL. Clinical epidemiology-a basic science for clinical medicine. Boston: Little Brown, 1991.

18 Gaydos CA, Howell MR, Quinn TC, et al. Use of ligase chain reaction with urine versus cervical culture for detection of Chlamydia trachomatis in an asymptomatic military population of pregnant and nonpregnant females attending Papanicolaou smear clinics. J Clin Microbiol 1998;36:1300-4.

19 Kacena KA, Quinn SB, Howell MR, et al. Pooling urine samples for ligase chain reaction screening for genital Chlamydia trachomatis infection in asymptomatic women. J Clin Microbiol $1998 ; 36: 481-5$

\section{Web top 10}

www.stijournal.com

These articles scored the most number of hits on STI's web site during January 2003

1 Cost effectiveness of screening for Chlamydia trachomatis: a review of published studies

E Honey, C Augood, A Templeton, I Russell, J Paavonen, P-A Mardh, A Stary, B Stray-Pedersen

December 2002;78: 406-12. (Review)

2 Brief Encounters

Mohsen Shahmanesh

December 2002;78:392.

3 Validation of a simplified grading of Gram stained vaginal smears for use in genitourinary medicine clinics

C A Ison, P E Hay

December 2002;78:413-15. (Diagnostics)

4 Chlamydia screening and sexual health

GJ Hart, B Duncan, K A Fenton

December 2002;78:396-7. (Editorial)

5 Behavioural surveillance: the value of national coordination

C A McGarrigle, K A Fenton, O N Gill, G Hughes,

D Morgan, BEvans

December 2002;78:398-405. (Review)

6 The female orgasm: a male perspective! Colm O'Mahony

December 2002;78:466. (Chester Chronicles)

7 National guideline for the management of suspected sexually transmitted infections in children and young people

A Thomas, G Forster, A Robinson, K Rogstad

October 2002;78:324-31. (Adolescent sexual health)

8 The potential role of suppressive therapy for sex partners in the prevention of neonatal herpes: a health economic analysis

R V Barnabas, H Carabin, G P Garnett

December 2002;78:425-9. (Original article)

9 Donovanosis

NO'Farrell

December 2002;78:452-7. (Tropical medicine)

10 Prevention interventions for HIV positive individuals

R J DiClemente, G M Wingood, C Del Rio, R A Crosby December 2002;78:393-5. (Editorial)

Visit the STI web site for hyperlinks to these articles, by clicking on "Top 10 papers"

www.stijournal.com 